Research Article

\title{
Reverse-Order Lower and Upper Functions for Periodic Problems of Second-Order Singular Difference Equations
}

\author{
Yanqiong Lu and Ruyun Ma \\ Department of Mathematics, Northwest Normal University, Lanzhou 730070, China \\ Correspondence should be addressed to Ruyun Ma; mary@nwnu.edu.cn
}

Received 22 May 2013; Accepted 9 September 2013

Academic Editor: Irena Rachůnková

Copyright (c) 2013 Y. Lu and R. Ma. This is an open access article distributed under the Creative Commons Attribution License, which permits unrestricted use, distribution, and reproduction in any medium, provided the original work is properly cited.

We present sufficient conditions ensuring the lower and upper functions on the reversed-order for the periodic difference equations. This enables us to obtain the existence of positive periodic solutions of the second-order difference equation $\Delta^{2} u(t-1)=g(t) / u^{\mu}(t)-$ $h(t) / u^{\lambda}(t)+f(t), t \in \mathbb{Z}$, where $g, h: \mathbb{Z} \rightarrow[0, \infty)$, and $f: \mathbb{Z} \rightarrow \mathbb{R}$ are $T$-periodic functions, $\lambda, \mu>0$.

\section{Introduction}

The theory of nonlinear difference equations has been widely used to study discrete models appearing in many fields such as computer science, economics, neural network, ecology, and cybernetics, see for example, [1]. Recently, there are many papers to study the existence of positive periodic solutions for second-order difference equations, see [2-7] and their references therein. However, there are few techniques for studying the existence of positive solutions of difference equations with singularity, and thus the results in the field are very rare, see [8-13]. The existence of positive periodic solutions for continuous case has been studied by Torres, see $[14,15]$.

Let $\mathbb{Z}$ denote the integer set, for $a, b \in \mathbb{Z}$ with $a<b$, $[a, b]_{\mathbb{Z}}:=\{a, a+1, \ldots, b\}$.

In 2012, Lu and $\mathrm{Ma}$ are concerned with the existence of positive periodic solutions of the second-order difference equation as follows:

$$
\Delta^{2} u(t-1)=\frac{g(t)}{u^{\mu}(t)}-\frac{h(t)}{u^{\lambda}(t)}+f(t), \quad t \in \mathbb{Z},
$$

where $g, h: \mathbb{Z} \rightarrow[0, \infty)$, and $f: \mathbb{Z} \rightarrow \mathbb{R}$ are $T$-periodic functions, $\lambda, \mu>0$. Special cases of (1) are

$$
\Delta^{2} u(t-1)=\frac{g(t)}{u^{\mu}(t)}-\frac{h(t)}{u^{\lambda}(t)}, \quad t \in \mathbb{Z},
$$

$$
\begin{aligned}
& \Delta^{2} u(t-1)=-\frac{h(t)}{u^{\lambda}(t)}+f(t), \quad t \in \mathbb{Z}, \\
& \Delta^{2} u(t-1)=\frac{g(t)}{u^{\mu}(t)}+f(t), \quad t \in \mathbb{Z} .
\end{aligned}
$$

In the related literature, it is said that (3) has an attractive singularity, whereas (4) has a repulsive singularity. They use the well-order lower and upper functions of (1) to show the existence of positive $T$-periodic solution of (1), (2), and (3), respectively, see [13].

However, the well-order lower and upper solutions lose their effects to deal with case (4). In this paper, we are devoted to constructing lower and upper functions on the reversedorder for (1) and dealing with the problems (1), (2), and (4), respectively.

The structure of the paper is as follows. Section 2 contains the tools needed in the proofs. In Section 3 we state and prove the main results and develop some corollaries for the equation with a singularity of mixed type. To illustrate the results, some examples are given.

\section{Auxiliary Results}

Let

$$
E:=\{u: \mathbb{Z} \longrightarrow \mathbb{R} \mid u(t)=u(t+T)\}
$$

under the norm $\|u\|=\max _{t \in[1, T]_{\mathbb{Z}}}|u(t)|$. Then $(E,\|\cdot\|)$ is a Banach space. 
The proofs of our results rely on the method of upper and lower functions. The following lemmas are classical and can be found, for example, in [3]. We introduce them in a form suitable for us.

Lemma 1. Let there exist positive functions $\alpha, \beta \in E$, such that

$$
\begin{array}{ll}
\Delta^{2} \alpha(t-1) \geq \frac{g(t)}{\alpha^{\mu}(t)}-\frac{h(t)}{\alpha^{\lambda}(t)}+f(t), \quad t \in \mathbb{Z}, \\
\Delta^{2} \beta(t-1) \leq \frac{g(t)}{\beta^{\mu}(t)}-\frac{h(t)}{\beta^{\lambda}(t)}+f(t), \quad t \in \mathbb{Z},
\end{array}
$$

and $\alpha(t) \leq \beta(t)$ for $t \in[1, T]_{\mathbb{Z}}$. Then there exists at least one positive $T$-periodic solution to (1).

A function $\alpha \in E$ (resp., $\beta \in E$ ) verifying (6) (resp, (7)) is called lower (resp, upper) function (solution) of (1). When the order between the lower and the upper functions is the inverse, an additional hypothesis is needed.

Definition 2. A function $\varphi \in E$ and $\varphi \geq 0$ is said to verify the property $(P)$ if the implication

$$
\begin{aligned}
u \in E & \Delta^{2} u(t-1)+\varphi(t) u(t) \geq 0, \quad t \in[1, T]_{\mathbb{Z}} \\
& \Longrightarrow u(t) \geq 0, \quad \text { for } t \in[1, T]_{\mathbb{Z}}
\end{aligned}
$$

holds.

Lemma 3. Let there exist positive functions $\alpha, \beta \in E$ satisfying (6), (7), and $\beta(t) \leq \alpha(t), t \in[1, T]_{\mathbb{Z}}$. Moreover, there exists $\varphi \in E$ with the property $(P)$, such that

$$
\frac{g(t)}{u^{\mu}(t)}-\frac{h(t)}{u^{\lambda}(t)}-\left(\frac{g(t)}{v^{\mu}(t)}-\frac{h(t)}{v^{\lambda}(t)}\right) \leq \varphi(t)[v(t)-u(t)],
$$$$
\text { for } t \in[1, T]_{\mathbb{Z}}
$$

where $\beta(t) \leq u(t) \leq v(t) \leq \alpha(t)$ for $t \in[0, T+1]_{\mathbb{Z}}$. Then there exists at least one positive T-periodic solution to (1).

Proof. From the condition (9), it follows that

$$
\frac{g(t)}{u^{\mu}(t)}-\frac{h(t)}{u^{\lambda}(t)}+\varphi(t) u(t) \leq \frac{g(t)}{v^{\mu}(t)}-\frac{h(t)}{v^{\lambda}(t)}+\varphi(t) v(t)
$$

That is, the nonlinearity is increasing.

Define the operator $T: E \rightarrow E$ as the unique solution of problem (1) as follows:

$$
T u(t)=\sum_{s=t+1}^{t+T} G(t, s)\left[\frac{g(s)}{u^{\mu}(s)}-\frac{h(s)}{u^{\lambda}(s)}+\varphi(t) u(t)+f(s)\right],
$$

where $G(t, s)$ is the Green's function of

$$
\Delta^{2} u(t-1)+\varphi(t) u(t)=0, \quad t \in \mathbb{Z}, u(t)=u(t+T) .
$$

As $\varphi$ satisfies the property $(P)$, it follows that $G(t, s)>0$. Now we divide the proof into three steps.

Step 1. We show

$$
T(K) \subset K,
$$

where $K=\{u \in E \mid \beta \leq u \leq \alpha\}$ is a nonempty bounded closed subset in $E$.

In fact, for $u \in K$, set $w=T u(t)$. From the definitions of $\alpha, \beta$, and $K$, combining with (9), we have

$$
\begin{gathered}
\Delta^{2}(w-\beta)(t-1)+\varphi(t)(w-\beta)(t) \geq 0, \\
(w-\beta)(t)=(w-\beta)(t+T) .
\end{gathered}
$$

Using property $(P)$, we get $\beta \leq w$.

Analogously, we can prove that $w \leq \alpha$. Thus, (13) holds.

Step 2. Let $u_{1}=T \eta_{1}, u_{2}=T \eta_{2}$, where $\eta_{1}, \eta_{2} \in K$ satisfy $\beta \leq \eta_{1} \leq \eta_{2} \leq \alpha$. Then we claim that

$$
u_{1} \leq u_{2} .
$$

In fact, let $\omega=u_{2}-u_{1}$, it follows from (10) and (11) that

$$
\begin{aligned}
\omega(t)= & T \eta_{2}-T \eta_{1} \\
=\sum_{s=t+1}^{t+T} G(t, s) & {\left[\frac{g(s)}{\eta_{2}^{\mu}(s)}-\frac{h(s)}{\eta_{2}^{\lambda}(s)}+\varphi(t) \eta_{2}(t)\right.} \\
& \left.\quad-\left(\frac{g(s)}{\eta_{1}^{\mu}(s)}-\frac{h(s)}{\eta_{1}^{\lambda}(s)}+\varphi(t) \eta_{1}(t)\right)\right] \geq 0 .
\end{aligned}
$$

Step 3. The sequences $\left\{\alpha_{n}\right\}$ and $\left\{\beta_{n}\right\}$ are obtained by recurrence:

$$
\begin{gathered}
\alpha_{0}=\alpha, \quad \beta_{0}=\beta, \\
\alpha_{n}=T \alpha_{n-1}, \quad \beta_{n}=T \beta_{n-1}, \quad n=1,2, \ldots .
\end{gathered}
$$

From the results of Steps 1 and 2, it follows that

$$
\beta=\beta_{0} \leq \beta_{1} \leq \cdots \leq \beta_{n} \leq \cdots \leq \alpha_{n} \leq \cdots \leq \alpha_{0}=\alpha .
$$

Moreover, from the definition of $T$, we get

$$
\begin{aligned}
& \Delta^{2} \alpha_{n}(t-1)+\varphi(t) \alpha_{n}(t) \\
& =\frac{g(t)}{\alpha_{n-1}^{\mu}(t)}-\frac{h(t)}{\alpha_{n-1}^{\lambda}(t)} \\
& \quad+\varphi(t) \alpha_{n-1}(t)+f(t), \quad t \in \mathbb{Z}, \\
& \alpha_{n}(t)=\alpha_{n}(t+T), \\
& \Delta^{2} \beta_{n}(t-1)+\varphi(t) \beta_{n}(t) \\
& =\frac{g(t)}{\beta_{n-1}^{\mu}(t)}-\frac{h(t)}{\beta_{n-1}^{\lambda}(t)} \\
& +\varphi(t) \beta_{n-1}(t)+f(t), \quad t \in \mathbb{Z}, \\
& \beta_{n}(t)=\beta_{n}(t+T) .
\end{aligned}
$$


This together with (18), we can easily get that there exists $C_{\alpha}$ depending only on $\alpha$ but not on $t$ and $n$, such that $\left|\alpha_{n}\right| \leq$ $C_{\alpha}$, so $\left\{\alpha_{n}\right\}$ is bounded in $E$. Similarly, $\left\{\beta_{n}\right\}$ is bounded in $E$. Therefore, we can conclude that $\left\{\alpha_{n}\right\}$ and $\left\{\beta_{n}\right\}$ converge uniformly to the extremal solutions $u$ of the problem (1). Subsequently, there exists at least one positive $T$-periodic solutions of (1).

Property $(P)$ is just an anti-maximum principle for the linear operator $L u:=\Delta^{2} u(t-1)+\varphi(t) u(t)$ with periodic boundary conditions, and it is equivalent to have a nonnegative Green function. Reference [7] provides sufficient conditions for $\varphi$ to verify property $(P)$. In particular, we have the following lemma.

Lemma 4 (see [7]). Let us assume $\varphi \in E, \varphi \not \equiv \quad 0$ and the following conditions holds:

$$
0 \leq \varphi(t)<4 \sin ^{2} \frac{\pi}{2 T}, \quad \text { for } t \in[1, T]_{\mathbb{Z}} .
$$

Then $\varphi$ verifies the property $(P)$.

To finish this section, we give a technical bound on the amplitude of oscillation of a periodic function.

Lemma 5 ([13, Lemma 2.2]). Given $v \in E$, then

$$
M_{v}-m_{v} \leq \frac{T}{4} \sum_{s=1}^{T}\left[\Delta^{2} v(s-1)\right]_{+}
$$

where

$$
\begin{aligned}
& M_{v}=\max \left\{v(t): t \in[1, T]_{\mathbb{Z}}\right\}, \\
& m_{v}=\min \left\{v(t): t \in[1, T]_{\mathbb{Z}}\right\} .
\end{aligned}
$$

Moreover, (21) is fulfilled as an equality if and only if $v$ is a constant function.

\section{The Main Results}

For the sake of brevity we will use the following notation throughout the paper:

$$
\begin{gathered}
G=\sum_{s=1}^{T} g(s), \quad H=\sum_{s=1}^{T} h(s), \quad F=\sum_{s=1}^{T} f(s), \\
F_{+}=\sum_{s=1}^{T}[f(s)]_{+}, \quad F_{-}=\sum_{s=1}^{T}[f(s)]_{-} .
\end{gathered}
$$

The following theorems are the main results of the paper.

Theorem 6. Let $G>0, F<0$, let functions $w, \sigma \in E$ be such that the equalities

$$
\begin{array}{ll}
\Delta^{2} w(t-1) & =H g(t)-G h(t), \quad \text { for } t \in \mathbb{Z}, \\
\Delta^{2} \sigma(t-1) & =\frac{|F|}{G} g(t)+f(t), \quad \text { for } t \in \mathbb{Z}
\end{array}
$$

are fulfilled and let there exist $x_{0} \in(0,+\infty)$ such that

$$
\begin{aligned}
& x_{0}\left(w(t)-m_{w}\right)+\sigma(t)-m_{\sigma} \\
& \quad \leq\left(\frac{G}{x_{0} G H+|F|}\right)^{1 / \mu}-\left(\frac{1}{x_{0} G}\right)^{1 / \lambda}, \quad t \in[1, T]_{\mathbb{Z}},
\end{aligned}
$$

where

$$
\begin{aligned}
& m_{w}=\min \left\{w(t): t \in[1, T]_{\mathbb{Z}}\right\}, \\
& m_{\sigma}=\min \left\{\sigma(t): t \in[1, T]_{\mathbb{Z}}\right\} .
\end{aligned}
$$

Moreover, define

$$
\begin{aligned}
\beta(t)= & \left(\frac{1}{x_{0} G}\right)^{1 / \lambda}+x_{0}\left(w(t)-m_{w}\right) \\
& +\sigma(t)-m_{\sigma}, \quad \text { for } t \in \mathbb{Z}
\end{aligned}
$$

and assume that $\varphi(t)=\mu g(t) / \beta^{1+\mu}(t)$ verifies the property $(P)$. Then problem (1) has at least one positive T-periodic solution.

Proof. Let $\beta$ be defined by (28). Then $\beta \in E$ and in view of (24) and (25), we have

$$
\begin{aligned}
\Delta^{2} \beta( & -1) \\
& =\left(x_{0} H+\frac{|F|}{G}\right) g(t)-x_{0} G h(t)+f(t), \quad \text { for } t \in \mathbb{Z} .
\end{aligned}
$$

Moreover, according to (26) and (27)

$$
\left(\frac{1}{x_{0} G}\right)^{1 / \lambda} \leq \beta(t) \leq\left(\frac{G}{x_{0} G H+|F|}\right)^{1 / \mu}, \quad \text { for } t \in \mathbb{Z} \text {. }
$$

Now (29) and (30) imply

$$
\Delta^{2} \beta(t-1) \leq \frac{g(t)}{\beta^{\mu}(t)}-\frac{h(t)}{\beta^{\lambda}(t)}+f(t), \quad \text { for } t \in \mathbb{Z} .
$$

Consequently, $\beta$ is an upper function to (1).

Further, we can choose $x_{1} \in\left(0, x_{0}\right)$ such that

$$
\begin{aligned}
& x_{1}\left(w(t)-m_{w}\right)+\sigma(t)-m_{\sigma} \\
& \quad \leq\left(\frac{1}{x_{1} G}\right)^{1 / \lambda}-\left(\frac{G}{x_{1} G H+|F|}\right)^{1 / \mu}, \quad \text { for } t \in[1, T]_{\mathbb{Z}}
\end{aligned}
$$

and put

$$
\begin{aligned}
\alpha(t)= & \left(\frac{G}{x_{1} G H+|F|}\right)^{1 / \mu}+x_{1}\left(w(t)-m_{w}\right) \\
& +\sigma(t)-m_{\sigma}, \quad \text { for } t \in \mathbb{Z} .
\end{aligned}
$$

Then $\alpha \in E$, and in view of (24) and (25) we have

$$
\Delta^{2} \alpha(t-1)=\left(x_{1} H+\frac{|F|}{G}\right) g(t)-x_{1} G h(t)+f(t), \quad t \in \mathbb{Z} .
$$


Moreover, according to (27) and (32)

$$
\left(\frac{G}{x_{0} G H+|F|}\right)^{1 / \mu} \leq \alpha(t) \leq\left(\frac{1}{x_{1} G}\right)^{1 / \lambda}, \quad \text { for } t \in \mathbb{Z} \text {. }
$$

Now (34) and (35) imply

$$
\Delta^{2} \alpha(t-1) \geq \frac{g(t)}{\alpha^{\mu}(t)}-\frac{h(t)}{\alpha^{\lambda}(t)}+f(t), \quad t \in \mathbb{Z}
$$

Consequently, $\alpha$ is a lower function to (1) and according to (30) and (35) we have

$$
\beta(t) \leq \alpha(t), \quad \text { for } t \in[1, T]_{\mathbb{Z}}
$$

Furthermore, note that the function

$$
\psi(y)=\frac{\mu}{\beta^{1+\mu}} y+\frac{1}{y^{\mu}}
$$

is nondecreasing for $y \geq \beta$. Therefore we have

$$
\begin{aligned}
& g(t)\left(\frac{\mu}{\beta^{1+\mu}(t)} u(t)+\frac{1}{u^{\mu}(t)}\right)-\frac{h(t)}{u^{\lambda}(t)} \\
& \quad \leq g(t)\left(\frac{\mu}{\beta^{1+\mu}(t)} v(t)+\frac{1}{v^{\mu}(t)}\right)-\frac{h(t)}{v^{\lambda}(t)}, \quad t \in[1, T]_{\mathbb{Z}},
\end{aligned}
$$

whenever $\beta(t) \leq u(t) \leq v(t)$ for $t \in[1, T]_{\mathbb{Z}}$, hence, we get

$$
\begin{aligned}
\frac{g(t)}{u^{\mu}(t)} & -\frac{h(t)}{u^{\lambda}(t)}-\left(\frac{g(t)}{v^{\mu}(t)}-\frac{h(t)}{v^{\lambda}(t)}\right) \\
& \leq \frac{\mu g(t)}{\beta^{1+\mu}(t)}(v(t)-u(t)), \quad \text { for } t \in[1, T]_{\mathbb{Z}} .
\end{aligned}
$$

Thus, the assertion follows from Lemma 3.

Remark 7. Note that for every $q \in E$ such that $\sum_{s=1}^{T} q(s)=0$, the periodic solution $v$ of the equation

$$
\begin{gathered}
\Delta^{2} v(t-1)=q(t), \quad \text { for } t \in[1, T]_{\mathbb{Z}}, \\
v(0)=v(T), \quad \Delta v(0)=\Delta v(T)
\end{gathered}
$$

is given by the Green formula:

$$
v(t)=c-\frac{1}{T}\left[\sum_{s=1}^{t-1}(T-t) s q(s)+\sum_{s=t}^{T} t(T-s) q(s)\right],
$$

where $c \in \mathbb{R}$. Therefore, the periodic functions $w$ and $\sigma$ with properties (24) and (25) exist and, moreover, are unique up to a constant term, the value of which has no influence on the validity of the condition (26). A similar observation can be made in relation to the formulations of the theorems given below.
Theorem 8. Let $\mu>\lambda, H>0, G>0, F=0$, let functions $w$, $\sigma \in E$ be such that (24) and (25) are fulfilled and let there exist $x_{0} \in(0,+\infty)$ such that

$$
\begin{aligned}
& x_{0}\left(w(t)-m_{w}\right)+\sigma(t)-m_{\sigma} \\
& \leq\left(\frac{1}{x_{0} H}\right)^{1 / \mu}-\left(\frac{1}{x_{0} G}\right)^{1 / \lambda}, \quad t \in[1, T]_{\mathbb{Z}}
\end{aligned}
$$

where $m_{w}$ and $m_{\sigma}$ are defined by (27). Moreover, assume that $\varphi(t)=\mu g(t) / \beta^{1+\mu}(t)$ verifies the property $(P)$, where $\beta$ is given by (28). Then problem (1) has at least one positive T-periodic solution.

Proof. Note that the inequality $\mu>\lambda$ implies

$$
\lim _{x \rightarrow 0^{+}}\left(\frac{1}{x G}\right)^{1 / \lambda}-\left(\frac{1}{x H}\right)^{1 / \mu}=+\infty
$$

Therefore, analogously to the proof of Theorem 6 , one can show that there exist lower and upper functions $\alpha, \beta$ satisfying (37). Consequently, the assertion follows from Lemma 3 with $\varphi(t)=\mu g(t) / \beta^{1+\mu}(t)$.

Corollary 9. Let $\mu>\lambda, H>0, G>0$, and let $w \in E$ be such that (24) is fulfilled. Let

$$
M_{w}-m_{w} \leq \frac{G^{(1+\mu) /(\mu-\lambda)}}{H^{(1+\lambda) /(\mu-\lambda)}}\left(\frac{(1+\mu) \lambda}{(1+\lambda) \mu}\right)^{((1+\mu) \lambda) /(\mu-\lambda)} \frac{\mu-\lambda}{(1+\lambda) \mu}
$$

where $m_{w}$ is given by (27) and

$$
M_{w}=\max \left\{w(t): t \in[1, T]_{\mathbb{Z}}\right\} .
$$

Moreover, let us define

$$
\begin{aligned}
\beta(t)= & \left(\frac{(1+\mu) \lambda}{(1+\lambda) \mu}\right)^{\mu /(\mu-\lambda)}\left(\frac{G}{H}\right)^{1 /(\mu-\lambda)}+\left(\frac{(1+\lambda) \mu}{(1+\mu) \lambda}\right)^{\lambda \mu /(\mu-\lambda)} \\
& \times \frac{H^{\lambda /(\mu-\lambda)}}{G^{\mu /(\mu-\lambda)}}\left(w(t)-m_{w}\right), \quad \text { for } t \in \mathbb{Z},
\end{aligned}
$$

and assume that $\varphi(t)=\mu g(t) / \beta^{1+\mu}(t)$ verifies the property $(P)$. Then problem (2) has at least one positive solution.

Proof. Put $f \equiv 0$ and

$$
x_{0}=\frac{H^{\lambda /(\mu-\lambda)}}{G^{\mu /(\mu-\lambda)}}\left(\frac{(1+\lambda) \mu}{(1+\mu) \lambda}\right)^{\lambda \mu /(\mu-\lambda)} .
$$

Then the assertion follows from Theorem 8.

Corollary 10. Let $G>0, F<0$, let $\sigma \in E$ be such that (25) is fulfilled, and let

$$
\left(M_{\sigma}-m_{\sigma}\right)^{\mu}|F|<G,
$$


where $m_{\sigma}$ is defined by (27) and

$$
M_{\sigma}=\max \left\{\sigma(t): t \in[1, T]_{\mathbb{Z}}\right\} .
$$

Moreover, if

$$
\frac{\mu|F|^{(1+\mu) / \mu} g(t)}{\left[G^{1 / \mu}-|F|^{1 / \mu}\left(M_{\sigma}-\sigma(t)\right)\right]^{1+\mu}}<4 \sin ^{2} \frac{\pi}{2 T}, \quad t \in[1, T]_{\mathbb{Z}}
$$

holds. Then problem (4) has at least one positive T-periodic solution.

Proof. Put $H=0$,

$$
x_{0}=\frac{|F|^{\lambda / \mu}}{G\left(G^{1 / \mu}-|F|^{1 / \mu}\left(M_{\sigma}-m_{\sigma}\right)\right)^{\lambda}}
$$

and define a function $\beta$ by (28). Let $\beta(t)=(G /|F|)^{1 / \mu}-$ $M_{\sigma}+\sigma(t)$ for $t \in[1, T]_{\mathbb{Z}}$. Then (51) guaranties that $\varphi(t)=$ $\mu g(t) / \beta^{1+\mu}(t)$ satisfies the property $(P)$. Moreover, (51) yields (25). Therefore, the assertion follows from Theorem 6.

Corollary 11. Let $G>0, F<0$ and

$$
\left(\frac{T}{4} \mu G\right)^{1 /(1+\mu)}|F|^{1 / \mu}+\frac{T}{4} F_{-}|F|^{1 / \mu} \leq G^{1 / \mu} .
$$

Then problem (4) has at least one positive T-periodic solution.

Proof. According to Lemma 5,

$$
M_{\sigma}-m_{\sigma} \leq \frac{T}{4} F_{-} .
$$

Then (53) implies (51). Consequently, the assertion follows from Corollary 10.

Example 12. Let us consider the boundary value problem:

$$
\begin{aligned}
& \Delta^{2} u(t-1)=\frac{1}{u}-\frac{1}{4 T}, \quad t \in[1, T]_{\mathbb{Z}}, \\
& u(0)=u(t), \quad \Delta u(0)=\Delta u(T),
\end{aligned}
$$

where $T>3$ is an integer.

Obviously, $(1 / u)-(1 / 4) \rightarrow+\infty$ as $u \rightarrow 0$. Let $g \equiv 1$, $f \equiv-1 / 4 T, \mu=1$. Then $G=T, F=-1 / 4$ and

$$
\left(\frac{T}{4} \mu G\right)^{1 /(1+\mu)}|F|^{1 / \mu}+\frac{T}{4} F_{-}|F|^{1 / \mu}=\frac{9 T}{64}<T=G^{1 / \mu} .
$$

Thus, from Corollary 11, problem (55) has at least one positive $T$-periodic solution.

\section{Acknowledgments}

The authors are very grateful to the anonymous referees for their valuable suggestions. This work was supported by the NSFC (no. 11061030, no. 11361054), Gansu provincial National Science Foundation of China (no. 1208RJZA258) and SRFDP (no. 20126203110004).

\section{References}

[1] W. G. Kelley and A. C. Peterson, Difference Equations, An Introduction with Applications, Academic Press, San Diego, Calif, USA, 1991.

[2] F. M. Atici and G. Sh. Guseinov, "Positive periodic solutions for nonlinear difference equations with periodic coefficients," Journal of Mathematical Analysis and Applications, vol. 232, no. 1, pp. 166-182, 1999.

[3] F. M. Atici and A. Cabada, "Existence and uniqueness results for discrete second-order periodic boundary value problems," Computers \& Mathematics with Applications, vol. 45, no. 6-9, pp. 1417-1427, 2003.

[4] F. M. Atici, A. Cabada, and V. Otero-Espinar, "Criteria for existence and nonexistence of positive solutions to a discrete periodic boundary value problem," Journal of Difference Equations and Applications, vol. 9, no. 9, pp. 765-775, 2003.

[5] T. He and $\mathrm{Y}$. Xu, "Positive solutions for nonlinear discrete second-order boundary value problems with parameter dependence," Journal of Mathematical Analysis and Applications, vol. 379, no. 2, pp. 627-636, 2011.

[6] R. Ma and H. Ma, "Positive solutions for nonlinear discrete periodic boundary value problems," Computers \& Mathematics with Applications, vol. 59, no. 1, pp. 136-141, 2010.

[7] R. Ma, Y. Lu, and T. Chen, "Existence of one-signed solutions of discrete second-order periodic boundary value problems," Abstract and Applied Analysis, vol. 2012, Article ID 437912, 13 pages, 2012.

[8] R. P. Agarwal and D. O'Regan, "Singular discrete boundary value problems," Applied Mathematics Letters, vol. 12, no. 4, pp. 127-131, 1999.

[9] R. P. Agarwal, K. Perera, and D. O’Regan, "Multiple positive solutions of singular and nonsingular discrete problems via variational methods," Nonlinear Analysis. Theory, Methods \& Applications, vol. 58, no. 1-2, pp. 69-73, 2004.

[10] H. Lv, D. O’Regan, and R. P. Agarwal, "Positive solution for singular discrete boundary value problem with sign-changing nonlinearities," Journal of Applied Mathematics and Stochastic Analysis, vol. 2006, Article ID 46287, 14 pages, 2006.

[11] I. Rachunková and L. Rachuneka, "Singular discrete problem arising in the theory of shallow membrane caps," Journal of Difference Equations and Applications, vol. 14, no. 7, pp. 747-767, 2008.

[12] R. Ma and Y. Lu, "Positive periodic solutions of secondorder difference equations with weak singularities," Advances in Difference Equations, vol. 2012, article 90, 2012.

[13] Y. Lu and R. Ma, "On positive periodic solutions of secondorder difference equations with attractive-repulsive singularities," Advances in Difference Equations, vol. 2012, article 186, 2012.

[14] P. J. Torres, "Weak singularities may help periodic solutions to exist," Journal of Differential Equations, vol. 232, no. 1, pp. 277284, 2007.

[15] R. Hakl and P. J. Torres, "On periodic solutions of second-order differential equations with attractive-repulsive singularities," Journal of Differential Equations, vol. 248, no. 1, pp. 111-126, 2010. 


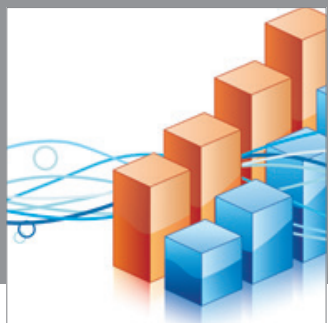

Advances in

Operations Research

mansans

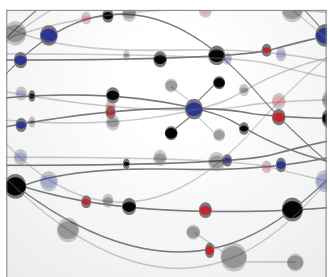

The Scientific World Journal
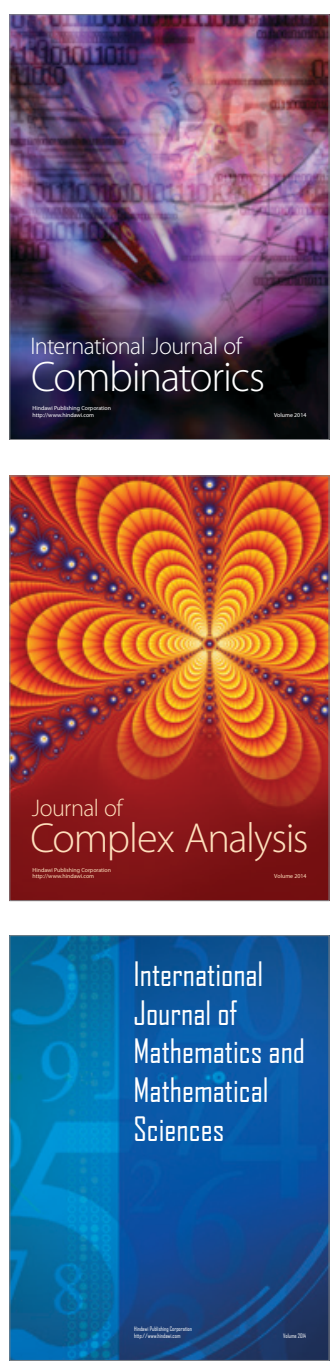
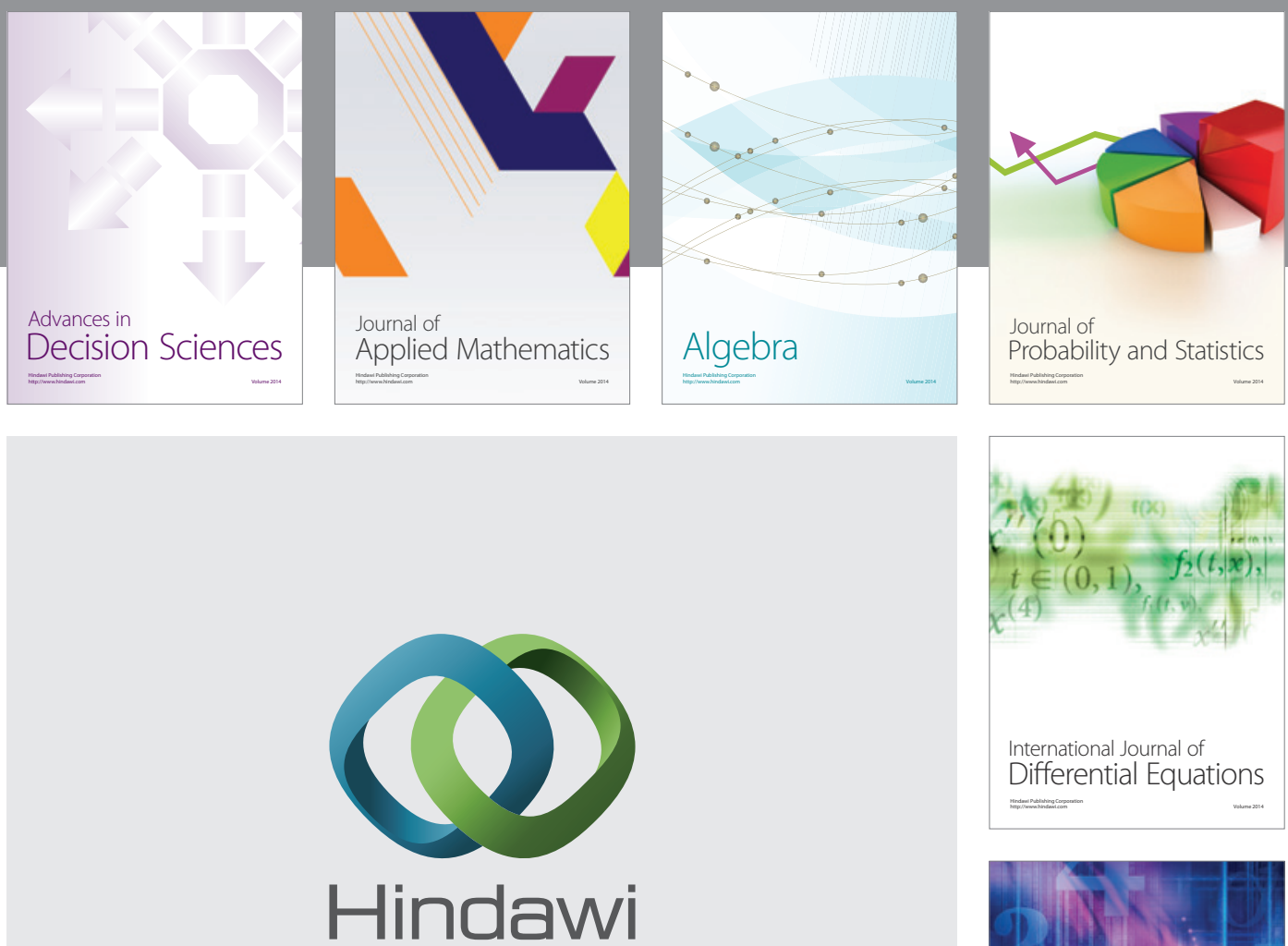

Submit your manuscripts at http://www.hindawi.com
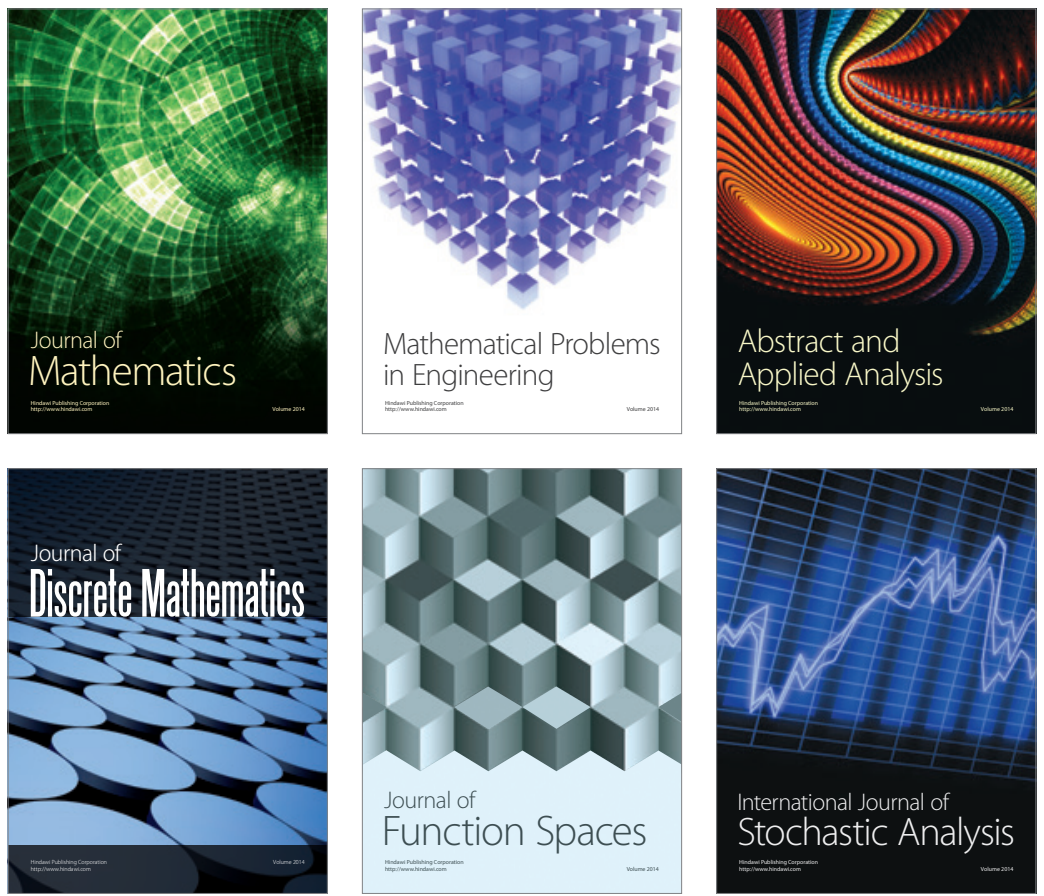

Journal of

Function Spaces

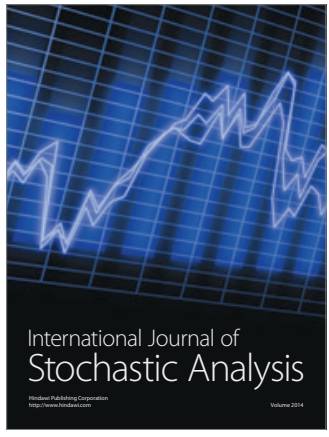

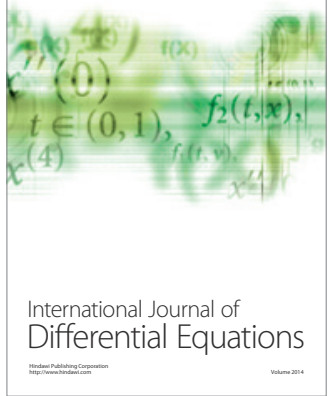
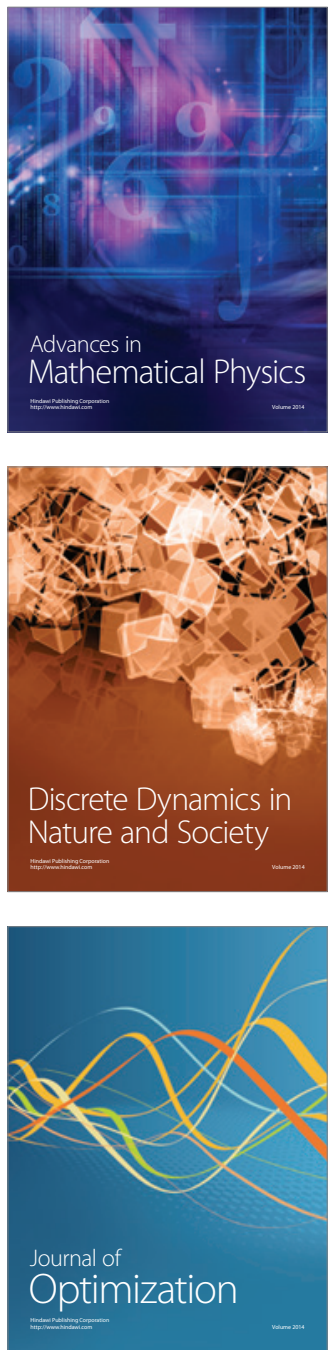